\title{
Analysis of Vitamin D Levels in Children with Progressive Myopia
}

\section{Sowmya Raveendra Murthy ${ }^{1 *}$ and Nitya Raghu ${ }^{2}$}

${ }^{1}$ Consultant, Department of Pediatric Ophthalmology and Strabismus, Sankara Eye

Hospital, Bengaluru, India

${ }^{2}$ DNB Student, Sankara Eye Hospital, Bengaluru, India

*Corresponding Author: Sowmya Raveendra Murthy, Consultant, Department of

Pediatric Ophthalmology and Strabismus, Sankara Eye Hospital, Bengaluru, India.
Received: April 26, 2021

Published: May 22, 2021

(C) All rights are reserved by Sowmya

Raveendra Murthy and Nitya Raghu.

\section{Abstract}

Background: The increasing prevalence of myopia in children today deserves more exploration into the causes and possible modifiable factors. With the growing prevalence of vitamin D deficiency in India, and its effect on numerous body systems, we attempted to find a possible association between vitamin D deficiency and progressive myopia.

Aim: To analyse the vitamin D levels in children with progressive myopia.

Methods: This is a pilot study. Children with progressive myopia on treatment with low dose atropine showing progression $(>0.5 \mathrm{D}$ increase in last 6 months) were included to be a part of the study. Few of these children showed progression despite being on low dose atropine which prompted us to search further. Serum levels of vitamin D3 levels were ordered and analyzed.

Results: Total of 20 children, 9 boys and 11 girls, were included, all in the age group of 11 to 17 years. Low dose atropine eye drops were being used for more than a year in 17 children and rest for past 5 months. Serum vitamin D 3 levels were noted to be deficient (< $20 \mathrm{ng} / \mathrm{ml}$ ) in 15 patients and insufficient (20 - 30 ng/ml) in 3 cases. Only 2 patients were noted to have normal levels above $30 \mathrm{ng} / \mathrm{ml}$.

Conclusion: Our pilot study throws light on possibly using vitamin D 3 levels in our protocols in treating children with progressive myopia.

Keywords: Progressive Myopia; Vitamin D Deficiency; Atropine Eye Drops

\section{Introduction}

Myopia is a common cause of visual impairment in children in the school going age group. The incidence of myopia has been estimated to be $5.3 \%$ in Indian children [1] and $35.6 \%$ in adults [2]. Needless to say, it is one of the most common causes of decrease in vision which starts manifesting in childhood and can progress over the years. Taking this into account, it becomes imperative to identify modifiable factors in the development and progression of myopia, so that progression can be minimized in childhood itself.

Known modifiable factors are time spent outdoors [3] versus time spent doing near work. More time spent outdoors is known to reduce the incidence or progression of myopia via a number of possible mechanisms: 1) light dopamine hypothesis, where on exposure to bright light or UV light more specifically, dopamine is released which modify axial length signaling pathways, 2) distant focus with relax of accommodation, 3) Increased Vitamin D levels [4]. The third mechanism i.e. vitamin $\mathrm{D}$ and its association with myopia has been under a lot of scrutiny.

With the emerging prevalence of myopia, research has aimed to find effective therapies to prevent further elongation of the globe to reduce the progression of myopia. One such therapy is the use of atropine drops in children with progressive myopia. The mechanism 
was initially assumed to be abolishing the excessive accommodation which triggered globe elongation. However, its efficacy in animal models after destruction of Edinger Westphal nucleus suggests that there is another mechanism involved [5].

Vitamin D is a fat soluble vitamin that also functions as a hormone. The most significant source of Vitamin D is synthesis in the skin on exposure to UV-B radiation. Other sources include dietary. It is equally possible that vitamin D levels and its association with myopia is a reflection of the effects of outdoor activities itself and is just a confounding factor [6]. However, a number of studies have looked into the same and identified Vitamin D deficiency as an independent risk factor, after removing outdoor work as a confounding factor [7]. India has a lower mean vitamin D level than most other countries despite having a tropical climate. The incidence of hypovitaminosis D in India has been estimated to be 30 to $91.2 \%$ in adults and 84.9 to $100 \%$ in children [8]. Hence it would be challenging but useful to study the possible association between vitamin $\mathrm{D}$ deficiency and myopia.

We have attempted a pilot study to analyse vitamin D levels in a group of school going Indian children with myopia, who have shown progression despite being on atropine eye drops.

\section{Materials and Methods}

Ethical clearance from the institutional review board was obtained. A group of children on regular follow up with myopia at Department of Pediatric ophthalmology in tertiary eye hospital, were found to have increasing myopic power despite being on Atropine $0.01 \%$ eye drops. This is a pilot study. Total of 20 children were selected and their serum samples sent for Vitamin D analysis. All of these children were on low dose atropine drops for control of progressive myopia. Low dose atropine was used for more than a year in 17 children and for past 5 months in 3 of them. Vitamin D was assessed by liquid chromatography tandem mass spectrometry. Levels above $20 \mathrm{ng} / \mathrm{ml}$ were labelled as adequate, below $20 \mathrm{mg} / \mathrm{ml}$ as deficient and below $10 \mathrm{ng} / \mathrm{ml}$ as severely deficient.

Data was collected pertaining to the refractive errors of the children and retrospective data of progression of their myopia over 6 months to 1 year, prior to vitamin $\mathrm{D}$ testing, was documented. The myopia was expressed as spherical equivalent of the refractive error and the change in refractive power was calculated.

\section{Results}

Out of the 20 children tested, vitamin D3 Levels noted to be deficient $(<20 \mathrm{ng} / \mathrm{ml})$ in 15 patients and insufficient $(20-30 \mathrm{ng} / \mathrm{ml})$ in 3 cases. Amongst those in the insufficient group, 6 of them had levels less than $10 \mathrm{ng} / \mathrm{dl}$. Two patients were found to have normal values of vitamin D3 (i.e. above $30 \mathrm{ng} / \mathrm{ml}$ ).

Out of the 15 children with deficiency, progression varied between -0.75 to $-2.62 \mathrm{D}$ (spherical equivalent). In the insufficient group, progression varied from -0.75 to $-2 \mathrm{D}$ and those with normal levels showed progression of -0.75 in 6 months.

\section{Discussion}

Studies outside India have proven Vitamin D as an independent risk factor in myopia $[9,10]$. Wilhelm., et al. [9] found that lower vitamin D levels was associated with larger axial lengths and higher risk of myopia. A study on a Korean population, who have a high prevalence of hypovitaminosis D like India, showed a higher risk of myopia among the deficient population. A metanalysis by Tang MS., et al. [7] showed that lower vitamin D concentrations were associated with a higher risk of myopia. However, they also inferred that this risk was more measurable in adults and was not significant in children. Although the mechanism of reduced myopia risk isn't known, one possible explanation is, vitamin D is known to influence dopamine metabolism in neurological disorders like Parkinson's disease. Hence it is a possibility that increased vitamin D serum levels increases dopamine secretion which is known to influence the axial length change via effect on gap junctions and receptive fields [9]. In a novel study by McMillan J [11], who studied the effects of Vitamin D on the eye, serial Pentacam images of the eyes of deficient children were taken. It was noticed that, among siblings, presence of vitamin D deficiency was associated with irregular astigmatism which also showed mild improvement on supplementation. In addition, the same was done on a pair of identical twins, one of whom spent most of her time outdoors and the other spending time indoors. Myopic progression was visible in the 'indoor twin' and not in the twin who played outdoors.

While the above studies did show a possible correlation, Specht., et al. [12] attempted to correlate Vitamin D levels of neonates with the development of myopia in young adulthood, in a case control study. Their results did not support the existence of a correlation. 
The only Indian study to look into the possible risk of vitamin D deficiency in myopia was by Annamaneni S., et al. [13] who tried to find a relation between vitamin D3 receptor (VDR) polymorphism and high myopia. However, they could not find any risk of myopia due to polymorphisms of the tested genes.

In our study as well, $75 \%$ of the children with progressive myopia had deficient levels of vitamin D. Emmetropisation results from a well-coordinated balance of multiple biological processes in the eye. One of the factors which influence accommodation in the eye is the ciliary muscle, which creates a clear retinal image of a close object. In the absence of accurate ciliary muscle action, e.g. mechanical factors, deficit in sympathetic stimulation, variation in the refractive status of components of the eye, there is a retinal blur and a possible trigger for axial length elongation. Another factor affecting ciliary muscle contraction is calcium homeostasis which is where Vitamin D can possibly come into play. However, as proven by Annamaneni., et al. [13] VDR gene did not play a direct role. Also, not all children who test deficient will have hypocalcemia.

Our aim was to understand the possibility of a treatable cause to myopia progression, and if increasing dose of atropine eye drops was warranted in such cases. With our current use of $0.01 \%$ eye drops having failed to arrest progression in some children, an increase to $0.05 \%$ or $0.1 \%$ may be required [14], however vitamin D involvement in the progression may change our plan of management.

Though $75 \%$ of the children with myopia presented with hypovitaminosis D, the high prevalence of vitamin D deficiency in Indian population is an important confounding factor. The parents of the children with vitamin D deficiency did claim that the child spent an hour for outdoor play every day though weren't sure of time of activity. However, the timing of outdoor play was not specified and this would also affect the vitamin D synthesis in the skin.

Also, our small sample size with inadequate number of control group of normal vitamin D levels is a limitation of this study.

\section{Conclusion}

Serum vitamin D3 levels were noted to be deficient in 75\% subset of children with progressive myopia. Considering vitamin D 3 levels are related to sunlight exposure, levels may act as surrogate marker for outdoor activities or act as independent factor in children with progressive myopia.
Our sample size is small to draw conclusions on association of progression with Vitamin D3 levels but definitely throws evidence of using vitamin D 3 levels in our protocols in treating children with progressive myopia.

This is a pilot study and we intend to note progression in children following vitamin D supplementation.

\section{Bibliography}

1. Sheeladevi S., et al. "Prevalence of Refractive Errors in Children in India: A Systematic Review". Clinical and Experimental optometry 101.4 (2018): 495-503.

2. Joseph S., et al. "Prevalence and risk factors for myopia and other refractive errors in an adult population in southern India". Ophthalmic and Physiological Optics 38.3 (2018): 346358.

3. Pan CW., et al. "Time Outdoors, Blood Vitamin D Status and Myopia: A Review". Photochemical and Photobiological Sciences 16.3 (2017): 426-432.

4. Kwon JW., et al. "Epidemiologic Survey Committee of the Korean Ophthalmological Society. Serum 25-hydroxyvitamin D level is associated with myopia in the Korea national health and nutrition examination survey". Medicine 95.46 (2016): e5012.

5. Chua WH., et al. "Atropine for the Treatment of Childhood Myopia”. Ophthalmology 113.12 (2006): 2285-2291.

6. Cuellar-Partida G., et al. "Genetically low vitamin D concentrations and myopic refractive error: a Mendelian randomization study". International Journal of Epidemiology 46.6 (2017): 1882-1890.

7. Tang SM., et al. "Vitamin D and its pathway genes in myopia: systematic review and meta-analysis". British Journal of Ophthalmology 103.1 (2019): 8-17.

8. Kamboj P., et al. "Prevalence of hypovitaminosis D in India and way forward". Indian Journal of Medical Research 148.5 (2018): 548-556.

9. Wilhelm JL., et al. "Low serum vitamin D is associated with axial length and risk of myopia in young children". European Journal of Epidemiology 31 (2016): 491-499.

10. Williams KM., et al. "Association Between Myopia, Ultraviolet B Radiation Exposure, Serum Vitamin D Concentrations, and Genetic Polymorphisms in Vitamin D Metabolic Pathways in a Multicountry European Study". JAMA Ophthalmology 135.1 (2017): 47-53. 
11. McMillan J. "Spectrum of Darkness, Agent of Light: Myopia, Keratoconus, Ocular Surface Disease, and Evidence for a Profoundly Vitamin D-dependent Eye". Cureus 10.6 (2018): e2744.

12. Specht IO., et al. "Neonatal vitamin D status and myopia in young adult men". Acta Ophthalmologica (2020).

13. Annamaneni S., et al. "Association of vitamin D receptor gene start codon (Fok1) polymorphism with high myopia". Oman Journal of Ophthalmology 4.2 (2011): 57-62.

14. Yam J C., et al. "Low-Concentration Atropine for Myopia Progression (LAMP) Study: A Randomized, Double-Blinded, Placebo-Controlled Trial of 0.05\%, 0.025\%, and 0.01\% Atropine Eye Drops in Myopia Control". Ophthalmology 126.1 (2019): 113-124.

Volume 5 Issue 6 June 2021

(C) All rights are reserved by Sowmya Raveendra Murthy and Nitya Raghu. 